\title{
Temperature-switchable surfactant-free microemulsion
}

Yongmin Zhang, Xuelian Chen, Bo Zhu, Yue Zhou, Xuefeng Liu,* and Cheng Yang

${ }^{\dagger}$ Key Laboratory of Synthetic and Biological Colloids, Ministry of Education, School of Chemical \& Materials Engineering, Jiangnan University, Wuxi 214122, P. R. China.

\section{CORRESPONDING AUTHOR: XFLIU@JIANGNAN.EDU.CN (X. LIU)}

Number of pages: 9

Number of figures: 19

Number of schemes:0

Number of tables: 0

Figure S1 Time-dependent self-correlation functions as obtained from DLS for the system n-butanol-DMSOwater. page S2

Figure S2 Snapshots of n-butanol-DMSO-water SFME as a beam of red light illuminates at the samples.....page $\mathrm{S}$

Figure S3 Snapshots of binary mixture page $\mathrm{S}$

Figure S4 Effects of water content on the UV-vis spectra of methyl orange in SFME at a fixed ratio of n-butanol to DMSO page S3

Figure S5 Effects of temperature on the area of single phase, pre-ouzo zone, and subregions. .page S4

Figure S6 The distribution of test points in ternary phase diagram and time-dependent self-correlation functions. page $\mathrm{S}$

Figure S7 Snapshots of formulations (that were selected from Figure S6A) after the addition of a small and same amount of water and n-butanol .page $\mathrm{S}$

Figure S8 Plotting the radius of the droplets for the formulation $a$ as a function of temperature page S5

Figure S9 Time-dependent self-correlation functions as obtained from DLS for the formulation $a$ ..page S

Figure S10 Snapshots of the formulation $b$ .page S

Figure S11 Snapshots of the formulation $c$ page $\mathrm{S}$

Figure 12 Size distribution of the formulation $b$. .page S7

Figure S13 Time-dependent self-correlation functions for the formulation $b$ page 
Figure S14 Time-dependent self-correlation functions for the formulation $c \ldots \ldots \ldots \ldots \ldots \ldots \ldots \ldots \ldots \ldots \ldots$.

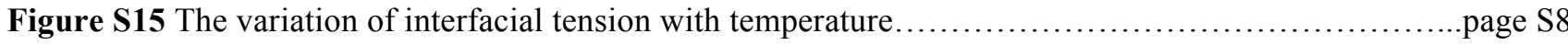

Figure S16 Snapshots of the formulation $c$ after the addition of $\mathrm{AgNO}_{3}$ and the system at $25{ }^{\circ} \mathrm{C}$ after separation of Ag-NPs-FME.

page S8

Figure S17 Size distribution of droplets of the formulation $c$

page S9

Figure S18 Size distribution of Ag nanoparticles obtained using n-butanol-DMSO-water SFME as a nanoreactor. page S9

Figure S19 Plotting the napierian logarithm of the absorbance of methyl orange at $400 \mathrm{~nm}$ as a function of time

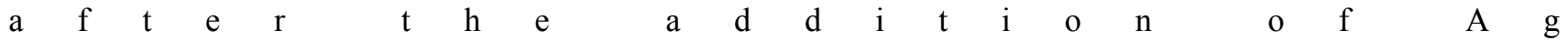
nanoparticles page S9

\section{Additional results}
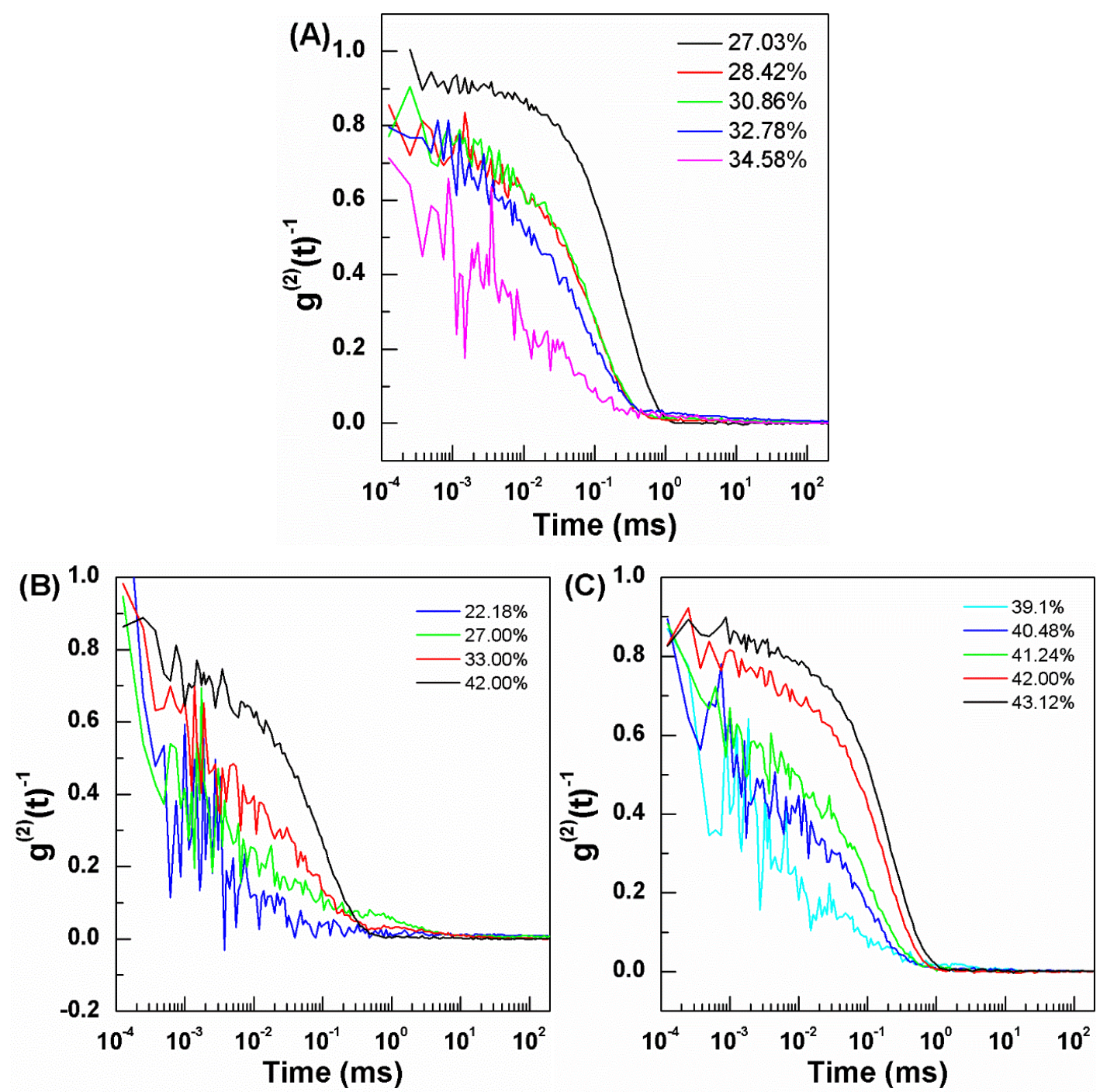
Figure S1 Time-dependent self-correlation functions as obtained from DLS for the system n-butanol-DMSO-water along the path indicated by (A) olive line (increasing DMSO at a fixed ratio of water to n-butanol), (B) cyan line (increasing n-butanol at a fixed ratio of DMSO to water) and (C) magenta line (increasing water at a fixed ratio of nbutanol to DMSO) in Figure 1.
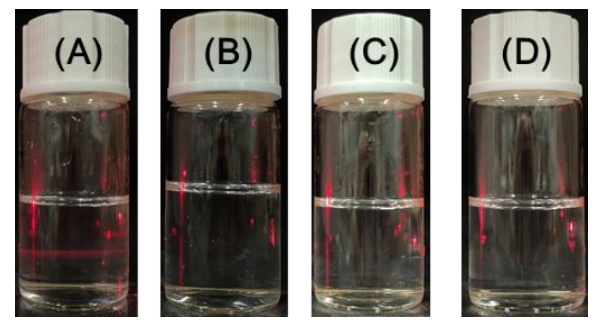

Figure S2 Snapshots of n-butanol-DMSO-water SFME as a beam of red light illuminates at the samples. (A) $37.00 \%$ n-butanol, 26.11\% DMSO and 36.89\% water; (B) 62.30\% n-butanol, $16.13 \%$ DMSO and $21.56 \%$ water; (C) $34.99 \%$ n-butanol, $35.30 \%$ DMSO and 29.72\% water and (D) $24.60 \%$ n-butanol, $39.77 \%$ DMSO and $35.63 \%$ water.

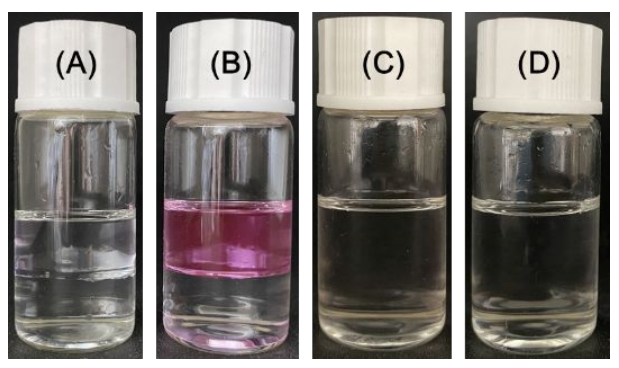

Figure S3 Snapshots of binary mixture: (A,B) water and n-butanol, (C) n-butanol and DMSO, and (D) water and DMSO. (B) was colored with nile red.

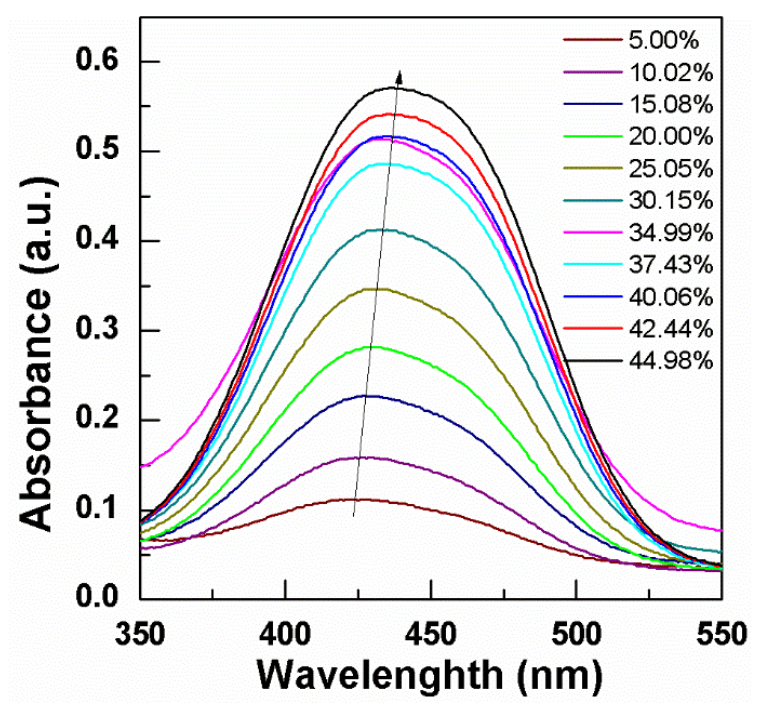

Figure S4 Effects of water content on the UV-vis spectra of methyl orange in SFME at a fixed ratio of n-butanol to DMSO. 

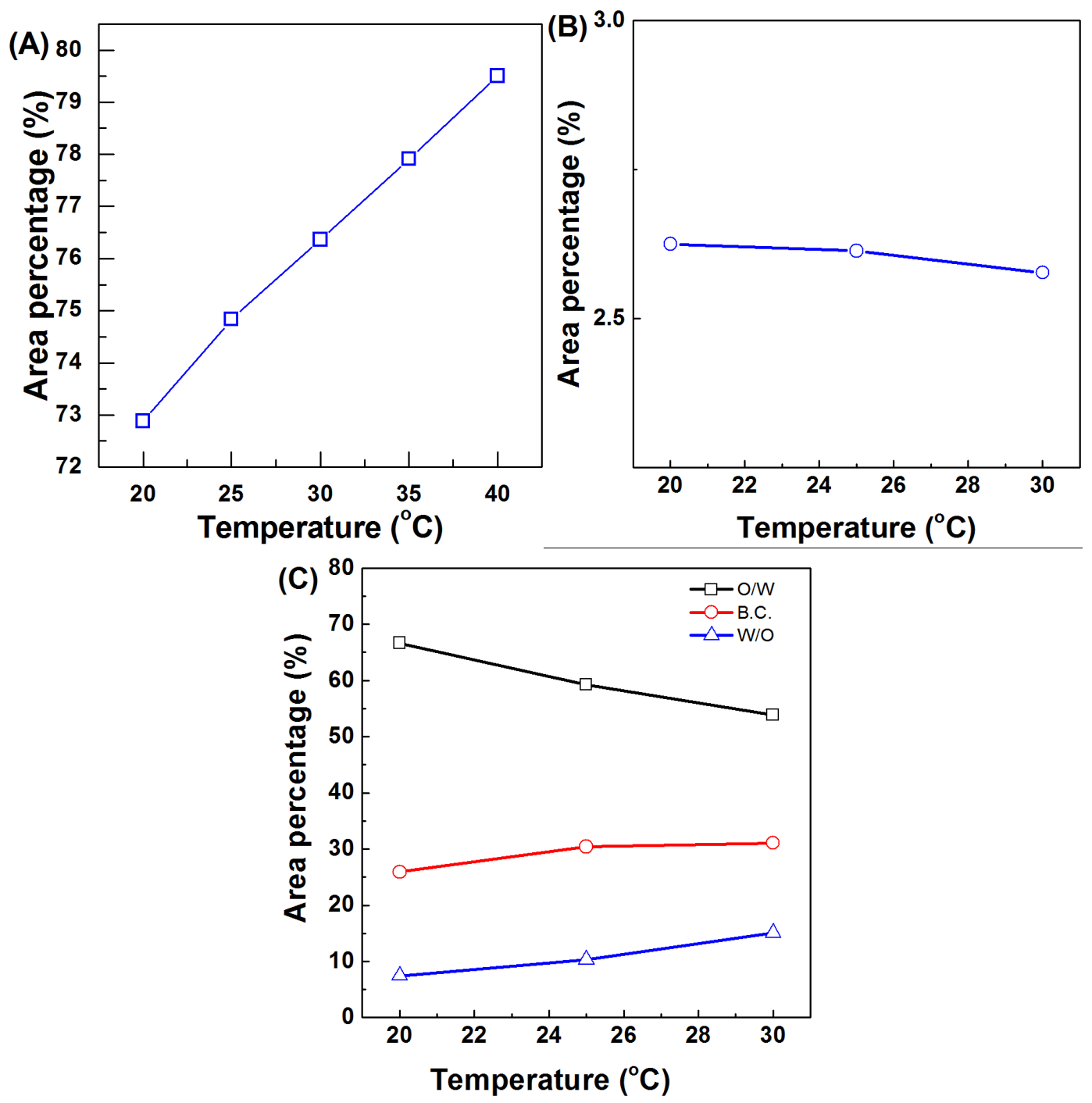

Figure S5 Effects of temperature on the area percentage of (A) single phase or (B) pre-ouzo zone to total area, and (C) subregions to the area of pre-ouzo zone.
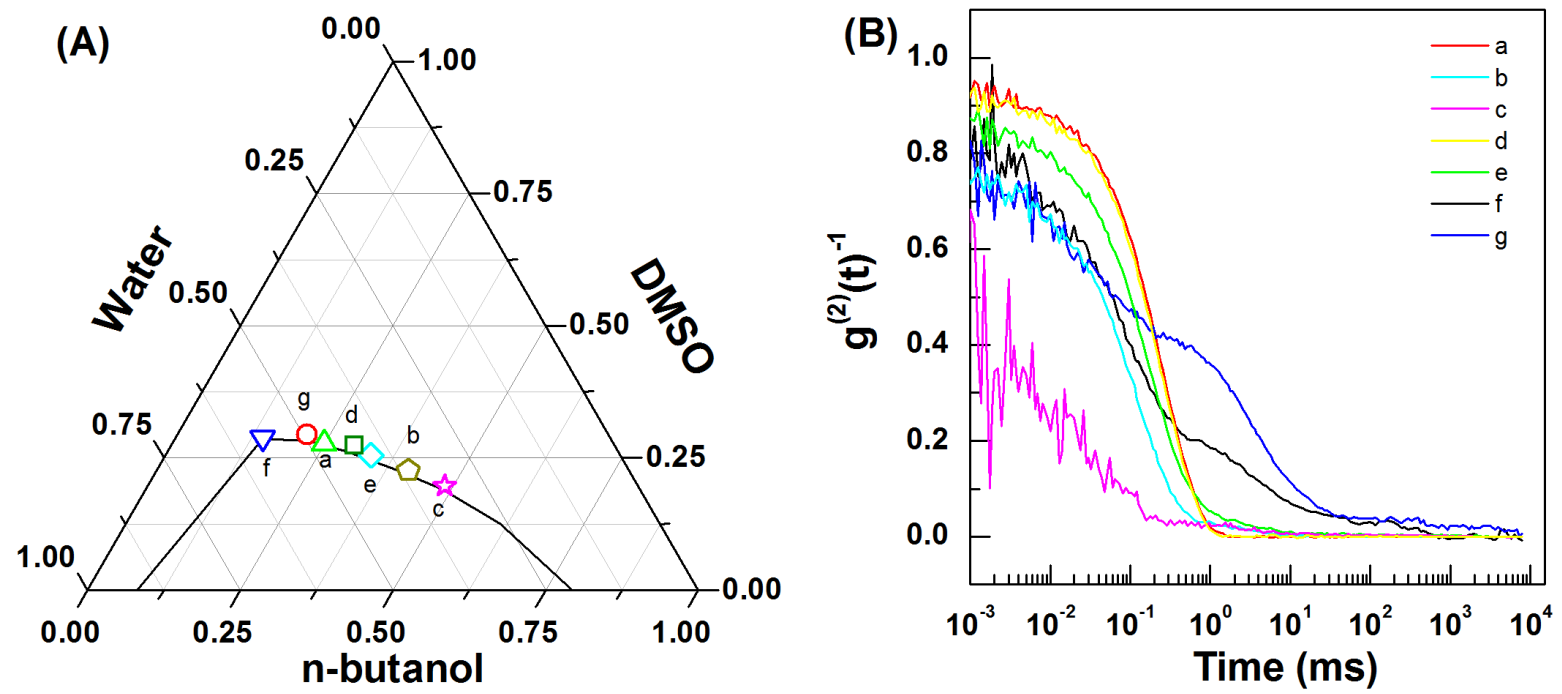

Figure S6 (A) The distribution of test points in ternary phase diagram and (B) Time-dependent self-correlation functions as obtained from DLS for the formulation selected from A. 


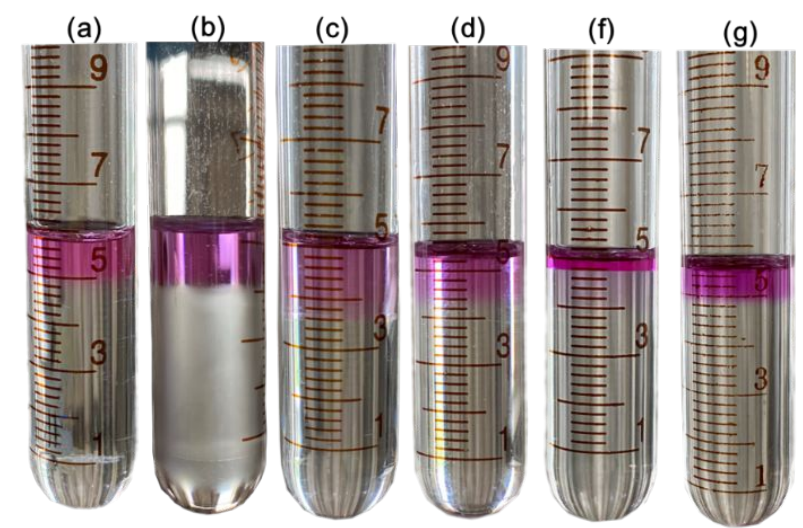

Figure S7 Snapshots of formulations (that were selected from Figure S6A) after the addition of a small and same amount of water and n-butanol at $25{ }^{\circ} \mathrm{C}$. The initial composition of formulations are (a) $24.80 \mathrm{wt} \% \mathrm{n}$-butanol, 27.77 wt $\%$ DMSO and water, (b) $41.31 \mathrm{wt} \%$ n-butanol, $22.62 \mathrm{wt} \%$ DMSO and water, (c) $48.73 \mathrm{wt} \% \mathrm{n}$-butanol, $19.66 \mathrm{wt} \%$ DMSO and water, (d) $30.02 \mathrm{wt} \% \mathrm{n}$-butanol, $27.37 \mathrm{wt} \%$ DMSO and water, (e) $33.71 \mathrm{wt} \% \mathrm{n}$-butanol, $25.52 \mathrm{wt} \%$ DMSO and water, (f) $14.33 \mathrm{wt} \% \mathrm{n}$-butanol, $28.78 \mathrm{wt} \%$ DMSO and water, (g) $21.28 \mathrm{wt} \% \mathrm{n}$-butanol, $29.43 \mathrm{wt} \%$ DMSO and water.

With the addition of a small and same amount of water and n-octanol, excessive n-butanol, or water, or both started to separate out. Be extra careful and careful when observing the interface, especially for the interface between $\mathrm{n}$ butanol and microemulsion. To make the interface clearer, n-butanol was colored with nile red. From Figure S7, one can see that in sample $\boldsymbol{a}$ the separated n-butanol and water have the same volume. Therefore, Extending the line between DMSO vertex and the coordinate point of original sample $\boldsymbol{a}$ to intersect the boundary of dexmion, yielding the critical point.

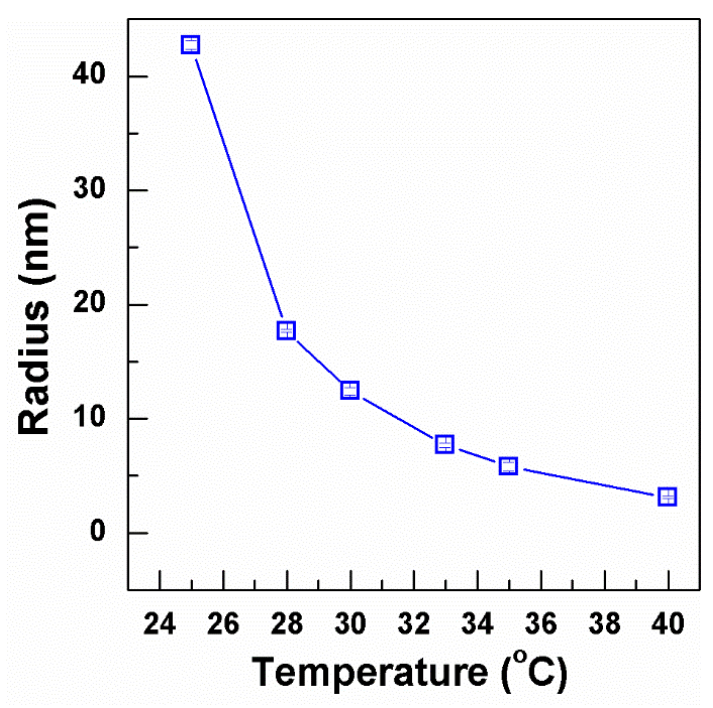

Figure S8 Plotting the radius of the droplets for the formulation $\boldsymbol{a}$ (43.78\% water, 29.19\% n-butanol and 27.03\% DMSO in O/W subregion indicated by a red star in Figure 1) as a function of temperature. 


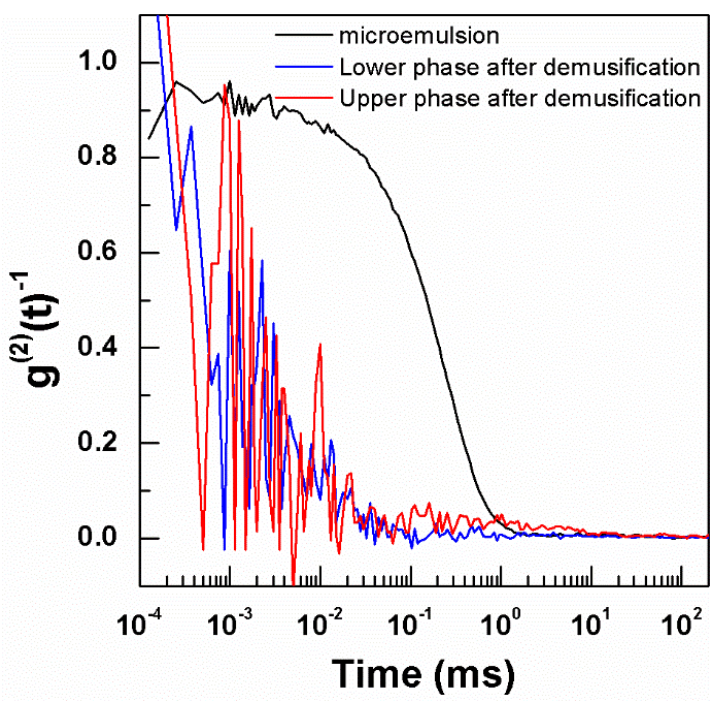

Figure S9 Time-dependent self-correlation functions as obtained from DLS for the formulation $\boldsymbol{a}$ (43.78\% water, 29.19\% n-butanol and 27.03\% DMSO) in O/W subregion indicated by a red star in Figure 1 before and after demulsification.

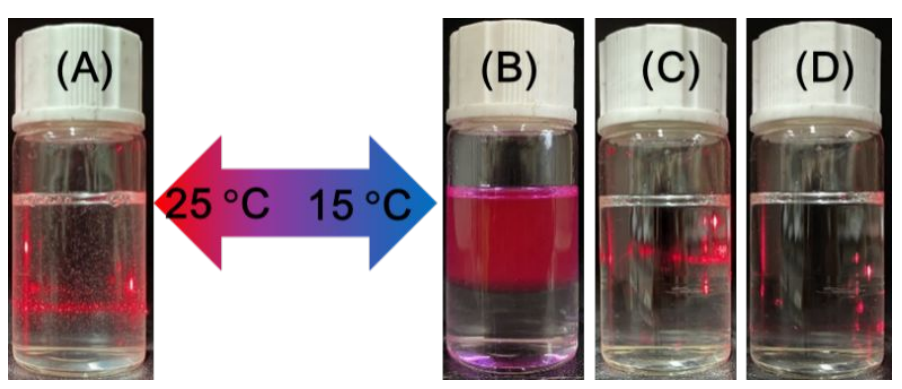

Figure S10 Snapshots of the formulation $\boldsymbol{b}$ (36.07\% water, 41.31\% n-butanol and 22.62\% DMSO) in BC subregion hinted by a blue circle in Figure 1 at (A) $25^{\circ} \mathrm{C}$ and (B-D) $15^{\circ} \mathrm{C}$. B was colored with oil-soluble nile red. A-C were illuminated by red laser light from the right.

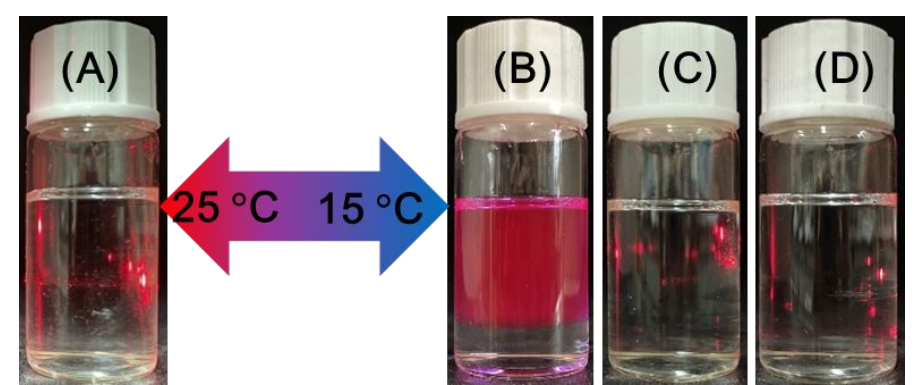

Figure S11 Snapshots of the formulation $c(29.66 \%$ water, $50.68 \% \mathrm{n}$-butanol and $19.66 \% \mathrm{DMSO})$ in W/O subregion indicated by an olive diamond in Figure 1 at (A) $25{ }^{\circ} \mathrm{C}$ and (B-D) $15{ }^{\circ} \mathrm{C}$. B was colored with oil-soluble nile red. A-C were illuminated by red laser light from the right. 


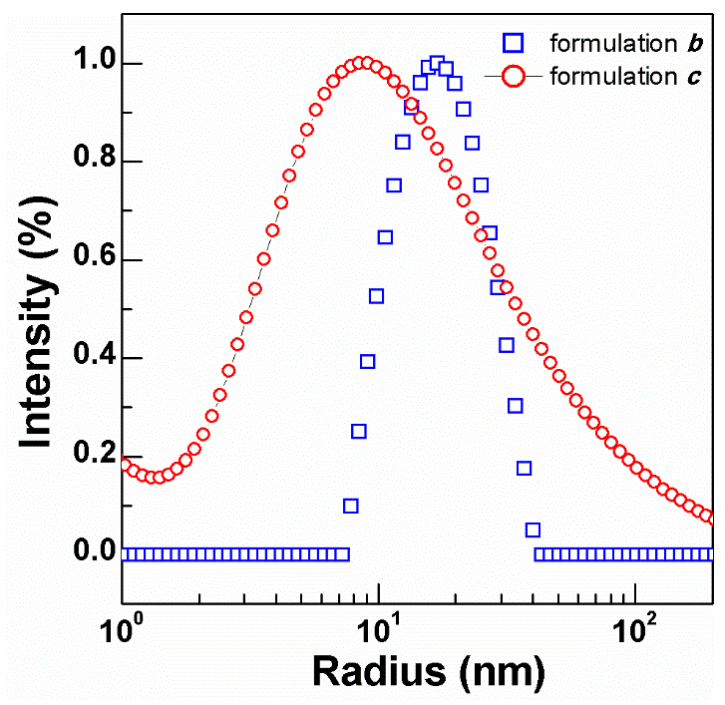

Figure 12 Size distribution of the formulation $\boldsymbol{b}$ (36.07\% water, $41.31 \%$ n-butanol and $22.62 \%$ DMSO in BC subregion hinted by a blue circle in Figure 1) and $c(29.66 \%$ water, $50.68 \%$ n-butanol and $19.66 \%$ DMSO in W/O subregion indicated by an olive diamond in Figure 1).

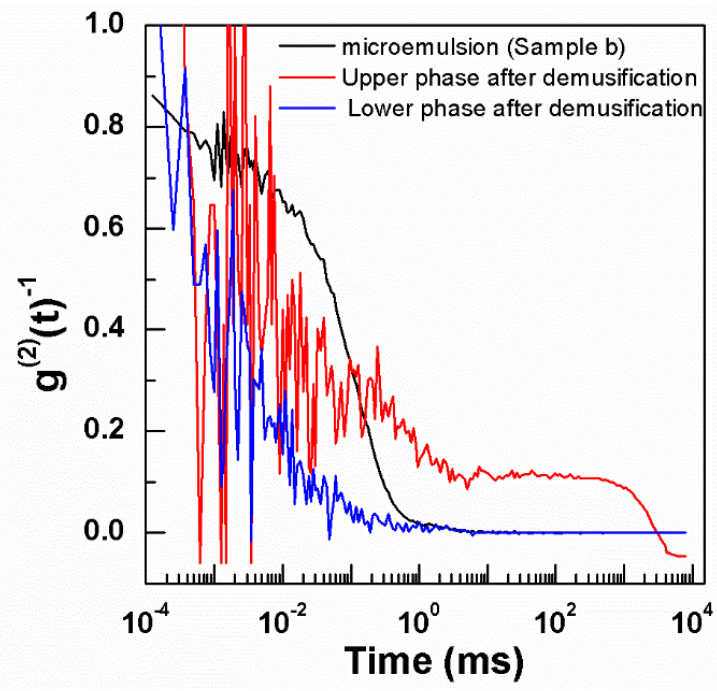

Figure S13 Time-dependent self-correlation functions as obtained from DLS for the formulation $\boldsymbol{b}$ (36.07\% water, $41.31 \%$ n-butanol and 22.62\% DMSO) in BC subregion hinted by a blue circle in Figure 1 before and after demulsification. 


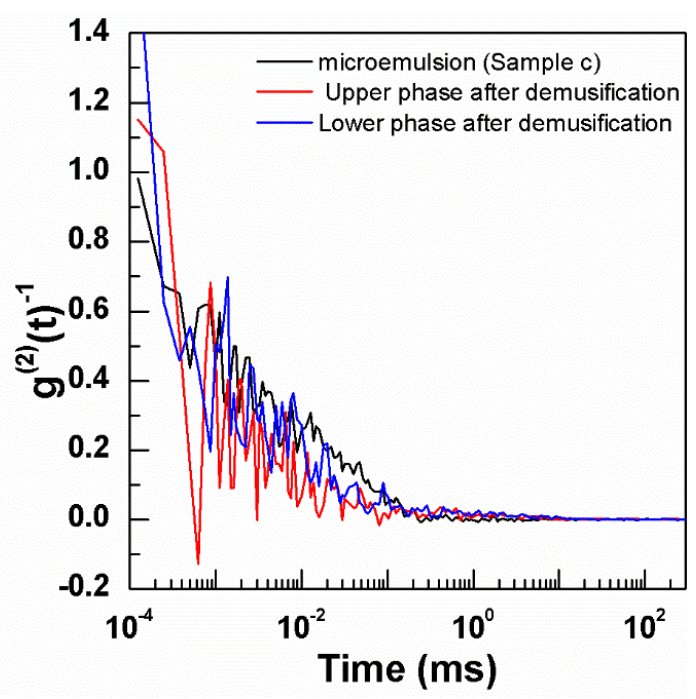

Figure S14 Time-dependent self-correlation functions as obtained from DLS for the formulation $c$ (29.66\% water, $50.68 \%$ n-butanol and 19.66\% DMSO) in W/O subregion indicated by an olive diamond in Figure 1 before and after demulsification.

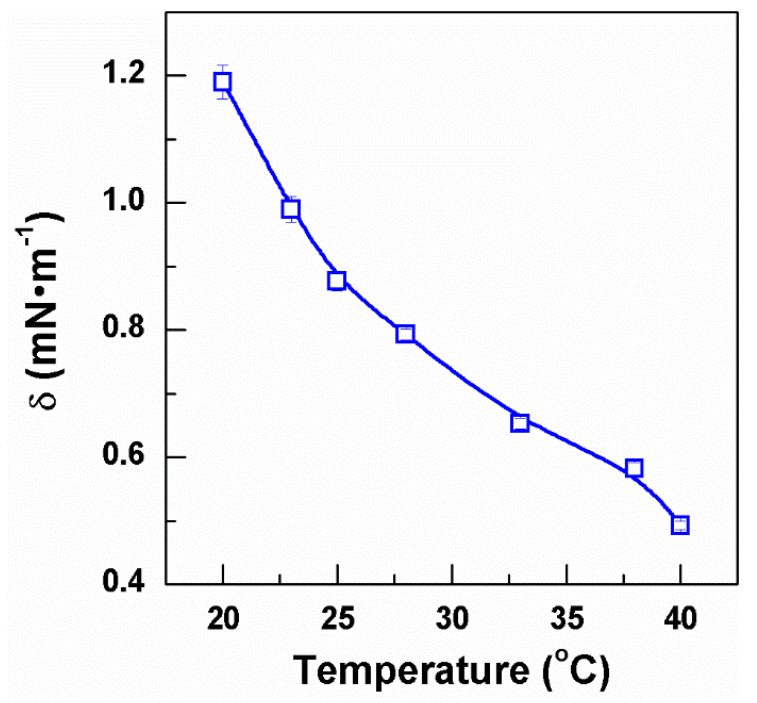

Figure S15 The variation of interfacial tension with temperature. The lower phase of the formulation $\boldsymbol{d}(35.22 \% \mathrm{n}$ butanol, $12.85 \%$ DMSO, and 51.93\% water) marked by a magenta triangle in Figure 1 was used as heavy phase, and the upper phase was used as light phase.

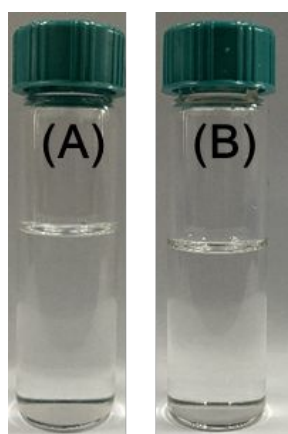

Figure S16 Snapshots of (A) the formulation $c$ (29.66\% water, 50.68\% n-butanol and $19.66 \%$ DMSO) after the addition of $\mathrm{AgNO}_{3}$ and (B) the system at $25^{\circ} \mathrm{C}$ after separation of Ag-NPs-SFME. 


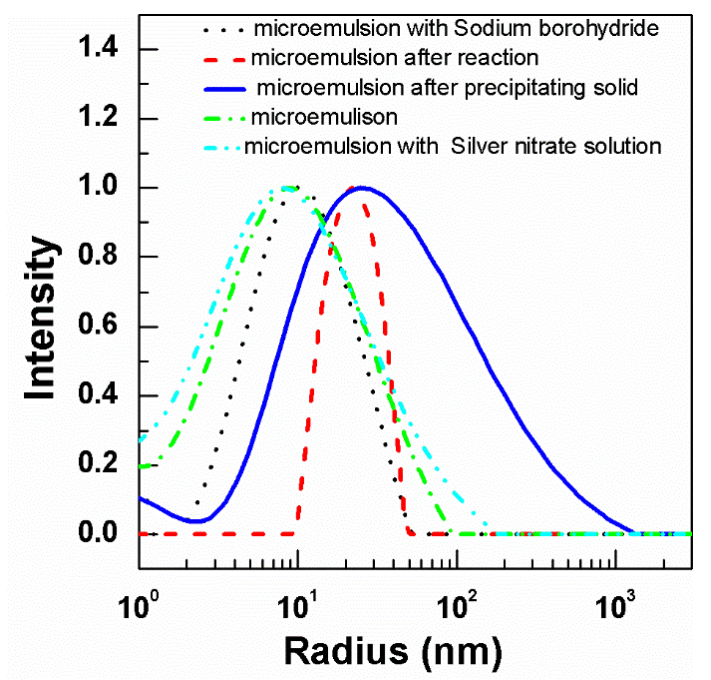

Figure S17 Size distribution of droplets of the formulation $\boldsymbol{c}$ (29.66\% water, 50.68\% n-butanol and 19.66\% DMSO) in W/O subregion indicated by an olive diamond in Figure 1.

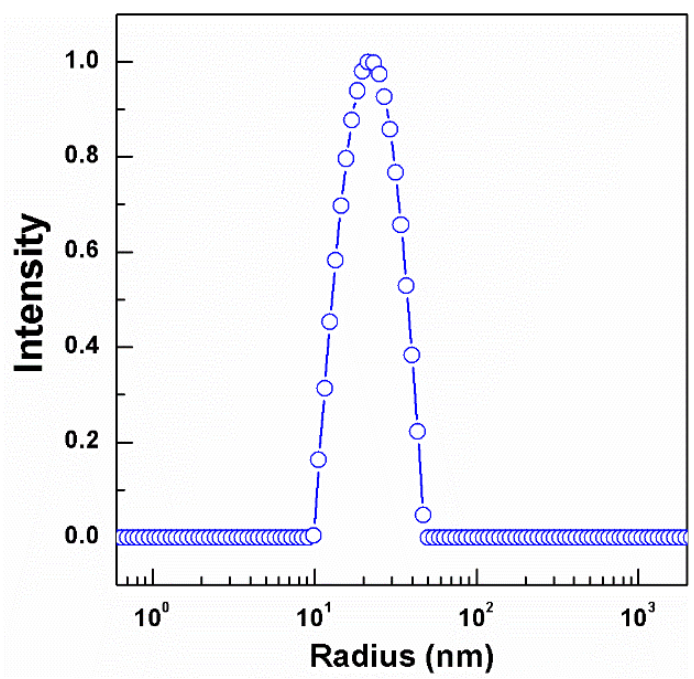

Figure S18 Size distribution of Ag nanoparticles obtained using n-butanol-DMSO-water SFME as a nano-reactor.

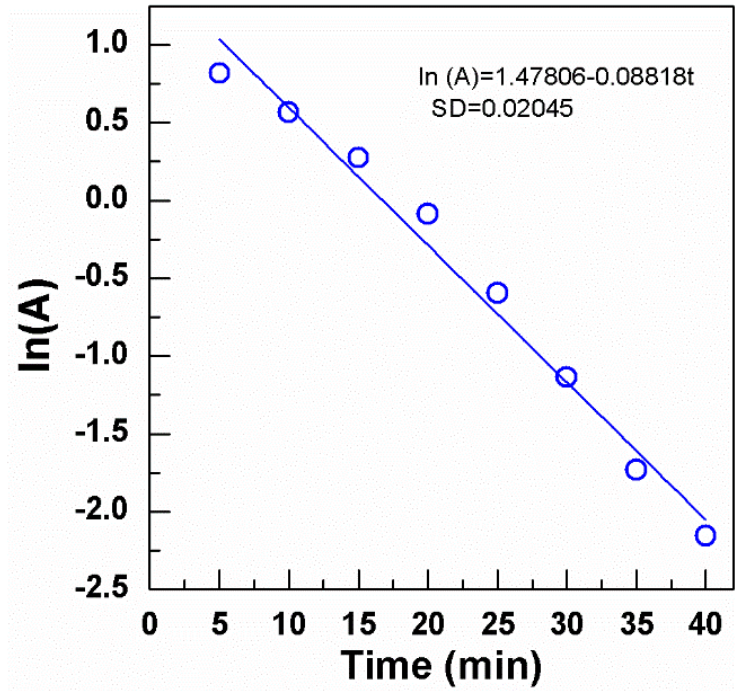

Figure S19 Plotting the napierian logarithm of the absorbance of methyl orange at $400 \mathrm{~nm}$ as a function of time after the addition of $\mathrm{Ag}$ nanoparticles. 